\title{
Nuclear Magnetic Resonance (NMR) Spectroscopy on the Effect of PCSK9 Inhibitor on Lipoprotein Particles in Patients With Acute Coronary Syndromes(ACS)
}

\section{tingting li}

Tianjin Medical University https://orcid.org/0000-0002-7901-8304

Hongliang Cong ( $\square$ hongliangcong@163.com )

Tianjin Medical University https://orcid.org/0000-0001-9288-7718

yingyi zhang

Tianjin Chest Hospital

\section{Research}

Keywords: PCSK9 Inhibitor, Nuclear Magnetic Resonance Spectroscopy, Lipoprotein Particles, Acute Coronary Syndromes, Atherosclerotic cardiovascular disease, low-density lipoprotein particle

Posted Date: August 24th, 2020

DOI: https://doi.org/10.21203/rs.3.rs-62828/v1

License: (c) (i) This work is licensed under a Creative Commons Attribution 4.0 International License.

Read Full License 


\section{Nuclear Magnetic Resonance (NMR) Spectroscopy on the Effect of PCSK9 Inhibitor on Lipoprotein Particles in Patients With Acute Coronary Syndromes(ACS)}

Tingting Li ${ }^{1}$, Yingyi Zhang ${ }^{2}$, Hongliang Cong ${ }^{2 *}$

1. Tianjin Medical University,Department of Cardiology, Tianjin Chest Hospital, Tianjin, China

2. Department of Cardiology,Tianjin Chest Hospital

Keywords: PCSK9 Inhibitor, Nuclear Magnetic Resonance Spectroscopy,Lipoprotein Particles,Acute Coronary Syndromes,Atherosclerotic cardiovascular disease, low-density lipoprotein particle 
1 Abstract

2 Objective: To assess the effects of proprotein convertase subtilisin/kexin type 9 (PCSK9) inhibitor (evolocumab) on blood lipid level, lipoprotein particles, and their subfractions with Nuclear Magnetic Resonance (NMR) spectroscopy in patients with acute coronary syndromes(ACS).

5 Methods: A total of 99 consecutive patients with ACS and poor lipid control were enrolled and assigned to either the experimental group $(n=54)$ or the control group $(n=45)$. The combination therapy of PCSK9 inhibitor (Repatha ${ }^{\circledR}, 140 \mathrm{mg}$, q2w) and moderate statin (rosuvastatin, $10 \mathrm{mg}$, qn) was administered in the experimental group, with moderate statin therapy (rosuvastatin, $10 \mathrm{mg}$, qn) alone in the control group. The therapeutic effects on blood lipid levels and lipoprotein particle subfractions were assessed with NMR spectroscopy after eight weeks of treatment, and the achievement of LDL-C treatment target in both groups was analyzed.

Results: In the experimental group, after eight weeks of evolocumab and moderate statin combination therapy, the level of blood lipids (TC, LDL-C and its subfractions [LDL-1 to 6], VLDL-C and its subfractions [VLDL-1 to 5], IDL-C, and HDL-C), lipoprotein particles, and their subfractions (VLDL-P, IDL-P, LDL-P, and its subfractions [LDL-P1 to 6], apoB, and LP(a)) demonstrated therapeutic benefits with statistical significance $(\mathrm{P}<0.05)$. Lowered level of LDL-P was attributed to the significant decrease of small LDL-P (LDL-P5+6), which was significantly more prominent than the decrease in medium LDL-P (LDL$\mathrm{P} 3+4)$ and large LDL-P $($ LDL-P1+2) $(\mathrm{P}<0.001)$. According to lipid control target recommended by the latest China Cholesterol Education Program (CCEP) Expert Consensus in 2019, the percentage of patients reaching the treatment target differed significantly between the experimental group and the control group (96.3\% and $13.3 \%$, respectively, $\mathrm{P}<0.001$ ).

Conclusions: PCSK9 inhibitor treatment for 8 weeks could significantly improve the plasma lipid profiles in ACS patients with poor lipid control, and significantly decrease the concentration of lipoprotein particles which could result in atherosclerosis. 


\section{Background}

2 Atherosclerotic cardiovascular disease (ASCVD) has posed grave threat to human health, while in cardiovascular diseases in China, accounting for $40 \%$ of disease deaths, presenting tremendous difficulties in prevention and treatment of the disease. Dyslipidemia, especially elevated levels of low-density lipoprotein cholesterol (LDL cholesterol, or LDL-C), is the major factor for ASCVD [12], making it the primary subject in the prevention and treatment of cardiovascular diseases to control blood lipids. In the Chinese Guidelines on Prevention and Treatment of Dyslipidemia in Adults issued in 2016, LDL-C was regarded as the most important indicator for early warning, medication modification, and lipid monitoring in ASCVD patients. However, in clinical practice, many individuals with normal or even low concentration of LDL-C $(<70 \mathrm{mg} / \mathrm{dL})$ experience ASCVD-related events or progression of atherosclerosis ${ }^{[3-4]}$. This residual risk indicates that a focus solely on the measurement of LDL-C is not an optimal strategy for all patients ${ }^{[5,6]}$. LDL is a heterogeneous lipoprotein fraction comprising different LDL subclasses that vary in size, density, and composition due to continuous remodeling of lipoproteins in the blood ${ }^{[7]}$, whose chemical components and physiologic functions differ a lot from each other. LDL particles of different sizes might not play the same role in the pathogenesis of ASCVD ${ }^{[8]}$, indicating that the size of LDL particles closely correlates with their functions, which is of grander clinical significance ${ }^{[9-11]}$.

Statins are the first choice for lipid-lowering drugs in clinical applications, which could also effectively decrease the risk of cardiovascular diseases. Nonetheless, studies revealed that even with high-dose statins, cardiovascular events were still of elevated incidence in high-risk patients. Furthermore, some patients show poor tolerance for high-dose statin therapy. In recent years, novel lipid-lowering medications, such as PCSK9 inhibitors, are receiving more attention, and impressive progresses were made in relevant studies. With the conclusion of a series of randomized clinical trials, the novel lipid-lowering medication, PCSK9 inhibitors, are gradually proven to be effective in lowering blood lipid levels and preventing cardiovascular diseases ${ }^{[12]}$.

This study aims to evaluate the effect of statins, the traditional lipid-lowering drug, on lipoprotein particles subfractions. In addition, the effect of PCSK9 inhibitor (Repatha $\left.{ }^{\circledR}\right)$, a novel lipid-lowering drug, on lipoprotein particles subfractions will also be explored. It has been demonstrated in a large number of studies that statins in combination with PCSK9 inhibitor can further decrease LDL-C level by $50 \%-70 \%{ }^{[13]}$, bringing greater cardiovascular benefit for patients. It remains unclear whether this benefit is attributed to an overall decrease in LDL-C or the decrease in certain lipoprotein subfractions, which would be further looked into in this study.

\section{Materials and Methods \\ 1.1 Study population}

This study was approved by the Medical Ethics Committee of Tianjin Chest Hospital. Informed consents were obtained from all subjects.ACS patients who presented to the Tianjin Chest Hospital from May to December 2019 were enrolled, with the inclusion criteria as follows: 1 . Informed consent obtained from the participant who voluntarily take the medications, and related documents signed; 2 . ACS patients who have a clearly documented LDL-C level higher than $2.6 \mathrm{mmol} / \mathrm{L}(100 \mathrm{mg} / \mathrm{dl})$ before taking any medication or an LDL-C level higher than $1.8 \mathrm{mmol} / \mathrm{L}(70 \mathrm{mg} / \mathrm{dl})$ while on lipid-lowering medications. The exclusion criteria include: 1 . Patients with severe primary cardiovascular or pulmonary diseases, as well as other serious diseases that could impair survival, or have conditions considered unfit for the study by researchers; 2. Metabolic or endocrine disorders (e.g. thyroid dysfunctions), abnormal coagulation, or major abnormalities in liver or renal function tests; 3. Pregnant or lactating women; 4. Suspected alcohol or other substance abuse, or other conditions that might impair follow-up or complicate subsequent treatment, deemed by the researcher, e.g. patients with frequent changes of workplace that might become lost on follow-up.

The 99 enrolled ACS patients with poor lipid control were assigned to two groups: 54 in the experimental group receiving the combination therapy of PCSK9 inhibitor (Repatha ${ }^{\circledR}, 140 \mathrm{mg}, \mathrm{q} 2 \mathrm{w}$ ) and moderate statin (rosuvastatin, $10 \mathrm{mg}$, qn), and 45 in the control group receiving moderate statin therapy (rosuvastatin, 10mg, qn) alone.

\subsection{Blood sample collection and lipid measurements}

At baseline and after 8 weeks of drug therapy, the participants in the two groups were collected peripheral venous blood at the fasting and resting state in the morning for examination . 
Routine blood lipid testing: The participants were fasted for 8 hours. The blood was collected in a serum tube containing an inert separating gel. After the blood was fully coagulated, it was centrifuged at $3000 \mathrm{rpm}$ for $10 \mathrm{~min}$, and the supernatant was taken for testing. The levels of plasma TC, HDL-C, LDL-C, and TG were measured by enzymatic measures using Roche c701 automated clinical chemistry analyzer. Apolipoprotein A1 (Apo-A1) and Apolipoprotein B (Apo-B) were measured by immunoturbidimetric methods. Lp(a) was measured by latex enhanced immuno-turbidimetry method.

Nuclear magnetic resonance (NMR) spectroscopy testing: $4 \mathrm{ml}$ of the participant's whole blood (fasting for 8 $\mathrm{h}$, BD blood vessels containing EDTA-K2 anticoagulant) was collected, centrifuged at $1500 \mathrm{~g}$ for $10 \mathrm{~min}$, and transferred the upper plasma into the cryopreserved tube, which was stored at $-80{ }^{\circ} \mathrm{C}$ for future testing. During the test, the samples were taken out of the refrigerator, and after thawing completely, $400 \mu 1$ plasma was taken and mixed with NMRS lipid buffer (Bruker Biospin, USA) 1:1, fully mixed, and then placed in a 5 mm NMR tube, and loaded into an automatic sample injector for testing ${ }^{[14]}$.

\subsection{Nuclear magnetic resonance (NMR) spectroscopy methods and testing program}

According to the standard operating procedure of AVANCE IVDr magnetic resonance spectrometer system( Bruker Biospin) ${ }^{[15,16]}$. The spectra were normalized to the same quantitative scale using Bruker's QuantRef manager within TopSpin which is based on the PULCON method; hence, the spectral intensity is normalized to proton concentration in units of millimoles per liter. For data analysis, the study selected the commercial Bruker IVDr LIpoprotein Subclass Analysis (B.I.-LISA) method ${ }^{[17,18]}$ as lipoprotein distribution prediction method, which used a PLS-2 regression model as the algorithm for spectral deconvolution ${ }^{[19]}$.This model provides information on main lipoprotein classes, including very low density lipoprotein (VLDL), intermediate density lipoprotein (IDL), LDL, and high density lipoprotein (HDL), as well as the six VLDL subclasses (VLDL-1 to VLDL-5), six LDL subclasses (LDL-1 to LDL-6), and four HDL subclasses (HDL-1 to HDL-4). Subclasses were sorted according to their increasing density and decreasing size in ascending order, respectively.

\subsection{Statistical analyses}

Statistical analyses were performed by SPSS 22.0. All the measurement data were presented as mean \pm standard deviation ( $\overline{\mathrm{x}} \pm \mathrm{s}$ ), and comparisons between groups were conducted with $\mathrm{T}$ test of independent samples. The enumeration data were presented as numbers (percentages), and comparisons between groups were conducted with Chi-square test. Skewed continuous variables were presented as median $\left(25^{\text {th }}\right.$ percentile, $75^{\text {th }}$ percentile), with comparisons between groups conducted by Mann-Whitney U test or Kruskal-Wallis test. Pearson correlation analysis was adopted when evaluating the consistency of lipid measurements by NMR spectroscopy and by routine blood lipid testing. Two-tailed P values $<0.05$ were considered statistically significant.

\section{Results}

\subsection{Baseline data}

The baseline data of the two groups was shown in Table 1

Table1. Baseline charateristics of the two groups and the comparisons of baseline blood lipid profiles and lipoprotein particles between them

\begin{tabular}{|l|l|l|}
\hline Variable & $\begin{array}{l}\text { PCSK9i+statins } \\
(\mathrm{n}=54)\end{array}$ & Statins $(\mathrm{n}=45)$ \\
\hline Age (years),mean \pm SD & $60.6 \pm 10.1$ & $58.6 \pm 10.6$ \\
\hline Men, $\mathrm{n}(\%)$ & $34(63.0 \%)$ & $30(66.7 \%)$ \\
\hline Hypertension, $\mathrm{n}(\%)$ & $32(59.3 \%)$ & $26(57.8 \%)$ \\
\hline Diabetes, $\mathrm{n}(\%)$ & $15(27.8 \%)$ & $10(22.2 \%)$ \\
\hline Smoking, $\mathrm{n}(\%)$ & $22(41.1 \%)$ & $23(51.1 \%)$ \\
\hline BMI $(\mathrm{kg} / \mathrm{m} 2))$, mean \pm SD & $26 \pm 5.0$ & $27 \pm 5.4$ \\
\hline STEMI,n(\%) & $10(18.5 \%)$ & $12(26.7 \%)$ \\
\hline NSTEMI,n(\%) & $24(44.4 \%)$ & $20(44.4 \%)$ \\
\hline UAP,n(\%) & $20(37.0 \%)$ & $13(28.9 \%)$ \\
\hline
\end{tabular}




\begin{tabular}{|l|l|l|}
\hline $\begin{array}{l}\text { Total cholesterol (mg/dL), mean } \pm \\
\text { SD }\end{array}$ & $214.2 \pm 42.4$ & $189.7 \pm 36.5$ \\
\hline LDL-C (mg/dL), mean \pm SD & $123.7 \pm 32.3$ & $103.7 \pm 28.1$ \\
\hline IDL-C (mg/dL), mean \pm SD & $15.5 \pm 9.1$ & $13.6 \pm 7.6$ \\
\hline VLDL-C (mg/dL), mean \pm SD & $31.9 \pm 17.9$ & $25.3 \pm 15.5$ \\
\hline HDL-C (mg/dL), mean \pm SD & $43.9 \pm 7.8$ & $44.3 \pm 9.4$ \\
\hline Lp(a)(nmol/l), median $(\mathrm{Q} 1, \mathrm{Q} 3) *$ & $73.1(13.7,102.3)$ & $32.7(8.2,49.1)$ \\
\hline ApoB $(\mathrm{mg} / \mathrm{dL})$, mean $\pm \mathrm{SD}$ & $108.7 \pm 24.2$ & $93.0 \pm 20.8$ \\
\hline LDL-P total (nmol/L), mean $\pm \mathrm{SD}$ & $1573.9 \pm 375.3$ & $1317.8 \pm 337.8$ \\
\hline VLDL-P(nmol/L), mean $\pm \mathrm{SD}$ & $237.5 \pm 106.0$ & $182.8 \pm 89.2$ \\
\hline IDL-P (nmol/L), mean \pm SD & $91.0 \pm 47.7$ & $88.6 \pm 43.3$ \\
\hline
\end{tabular}

Abbreviations: BMI= Body Mass Index ;STEMI =Stsegment Elevation Myocardial Infarction ;NSTEMI =non-ST-segment elevation myocardial infarction ; UAP $=$ Unstable Angina Pectoris;LDL-C $=$ low-density lipoprotein cholesterol;IDL-C=intermediate-density lipoprotein;VLDL-C = very low-density lipoprotein cholesterol; HDL-C = high-density lipoprotein cholesterol; Lp(a)=Lipoprotein a; ApoB $=$ Apolipoprotein $\mathrm{B} ; \mathrm{LDL}-\mathrm{P}=$ low-density lipoprotein particle concentration;VLDL-P = very low-density lipoprotein particle concentration;IDL-P = intermediate-density lipoprotein particle concentration; Q1, Q3 = fifirst and third quartiles; SD = standard deviation

Demographic data, clinical characteristics, baseline blood lipid profiles, and lipoprotein particle concentrations of the 99 patients were shown in Table $1.63 .0 \%$ and $66.7 \%$ were males in the experimental group and control group, respectively; while $27.8 \%$ and $22.2 \%$ were diabetic, respectively. No significant difference was found in age, gender, comorbidities including hypertension and diabetes mellitus, and body mass index (BMI) $(\mathrm{P}>0.05)$ between the two groups. The comparisons of the prevalence of ST-elevation myocardial infarction (STEMI), non-ST segment elevation myocardial infarction (NSTEMI), and unstable angina pectoris (UAP) revealed no significant difference $(\mathrm{P}>0.05)$. At baseline, the median and interquartile range of LP(a) levels in the two groups were73.1 $(13.7,102.3)$ and $32.7(8.2,49.1)$, respectively, significantly higher in the experimental group with a $\mathrm{P}$ value of 0.032 . Other baseline lipid and lipoprotein particle profiles were comparable between the two groups. The LDL-C levels in the experimental and control groups were $123.7 \pm 32.3 \mathrm{mg} / \mathrm{dL}$ and $103.7 \pm 28.1 \mathrm{mg} / \mathrm{dL}$, respectively;and LDL-P levels were $1573.9 \pm$ $375.3 \mathrm{nmol} / \mathrm{L}$ and $1317.8 \pm 337.8 \mathrm{nmol} / \mathrm{L}$, respectively. Other baseline characteristics of blood lipid profiles and lipoprotein particles were shown in Table 1.

\subsection{Correlations between NMR spectroscopy and enzymatic method results in the measurement of lipid parameters}

Pearson correlation analyses were performed to assess the consistency between the NMR spectroscopy and enzymatic method tests, in terms of the six items that they had in common (triglycerides [TG], total cholesterol [TC], LDL-C, high-density lipoprotein [HDL-C], Apo-A1, and Apo-B). The correlation coefficient was between 0.839 and 0.912 , indicating close correlation between the two sets of test.

Table 2. Pearson coefficient of correlation in lipid parameters measured by NMR spectroscopy and enzymatic method

\begin{tabular}{|l|l|l|}
\hline & R values & P values \\
\hline TG & 0.849 & 0.000 \\
\hline TC & 0.912 & 0.000 \\
\hline HDL-C & 0.858 & 0.000 \\
\hline LDL-C & 0.839 & 0.000 \\
\hline Apoa1 & 0.865 & 0.000 \\
\hline ApoB & 0.854 & 0.000 \\
\hline
\end{tabular}

\subsection{Changes in lipid profiles after treatment in the two groups}

Table 3. Absolute and relative reduction in lipid levels and lipoprotein particle concentration compared to baseline after eight weeks of treatment 


\begin{tabular}{|c|c|c|c|c|c|c|}
\hline & \multicolumn{3}{|c|}{ PCSK9i+statins } & \multicolumn{3}{|c|}{ statins } \\
\hline & Week8 & $\begin{array}{l}\text { Change from } \\
\text { baseline }\end{array}$ & $\begin{array}{l}\text { Percent } \\
\text { change } \\
\text { from } \\
\text { baseline } \\
(\%)\end{array}$ & Week8 & $\begin{array}{l}\text { Change from } \\
\text { baseline }\end{array}$ & $\begin{array}{l}\text { Percent } \\
\text { change } \\
\text { from } \\
\text { baseline } \\
(\%)\end{array}$ \\
\hline $\begin{array}{l}\text { VLDL-C } \\
\text { (mg/dl) } \\
\text { Total }\end{array}$ & $17.2 \pm 7.3$ & $14.7 \pm 15.4$ & 26 & $20.0 \pm 12.1$ & $5.2 \pm 12.9$ & 7.9 \\
\hline VLDL1 & $6.7 \pm 4.6$ & $6.1 \pm 9.1$ & 26.3 & $6.5 \pm 4.5$ & $3.5 \pm 6.6$ & 21.6 \\
\hline VLDL2+3 & $2.6 \pm 1.5$ & $2.8 \pm 2.7$ & 20.4 & $3.6 \pm 2.5$ & $0.6 \pm 2.3$ & -20 \\
\hline VLDL4+5 & $1.9 \pm 1.3$ & $2.2 \pm 2.5$ & 40.9 & $2.9 \pm 2.3$ & $0.6 \pm 1.7$ & 2.8 \\
\hline IDL-C (mg/dl) & $5.7 \pm 4.1$ & $10.0 \pm 7.9$ & 62.0 & $9.3 \pm 4.7$ & $4.3 \pm 6.4$ & 20.7 \\
\hline $\begin{array}{l}\text { LDL-C } \\
\text { (mg/dl) } \\
\text { Total } \\
\end{array}$ & $43.8 \pm 21.8$ & $81.6 \pm 28.7$ & 65.3 & $80.7 \pm 14.8$ & $22.9 \pm 25.1$ & 18.1 \\
\hline $\mathrm{LDL}_{1+2}$ & $10.4 \pm 4.3$ & $9.6 \pm 8.5$ & 40.4 & $11.3 \pm 4.6$ & $4.2 \pm 5.7$ & 21.2 \\
\hline $\mathrm{LDL}_{3+4}$ & $7.0 \pm 4.9$ & $10.2 \pm 9.4$ & 50.3 & $11.1 \pm 4.5$ & $2.4 \pm 5.9$ & -20.7 \\
\hline LDL5+6 & $5.0 \pm 5.6$ & $21.2 \pm 12.7$ & 79.5 & $17.6 \pm 5.9$ & $5.4 \pm 7.4$ & 6.6 \\
\hline $\begin{array}{l}\text { HDL-C } \\
(\mathrm{mg} / \mathrm{dl}) \\
\text { Total } \\
\end{array}$ & $46.9 \pm 8.3$ & $-2.9 \pm 6.5$ & -7.6 & $44.8 \pm 8.3$ & $-0.4 \pm 9.0$ & -3.4 \\
\hline $\mathrm{HDL}_{1+2}$ & $10.2 \pm 4.4$ & $0.7 \pm 3.6$ & -2.2 & $10.5 \pm 4.2$ & $0.1 \pm 2.6$ & -2.7 \\
\hline $\mathrm{HDL}_{3+4}$ & $12.6 \pm 5.4$ & $-1.9 \pm 3.2$ & -82.7 & $11.3 \pm 5.3$ & $-0.6 \pm 4.1$ & 27.2 \\
\hline $\mathrm{TC}(\mathrm{mg} / \mathrm{dl})$ & $109.9 \pm 27.9$ & $106.0 \pm 37.8$ & 48.4 & $153.7 \pm 18.4$ & $35.9 \pm 33.7$ & 16.9 \\
\hline $\begin{array}{l}\text { VLDL-P } \\
(\mathrm{nmol} / \mathrm{l})\end{array}$ & $158.2 \pm 56.9$ & $80.2 \pm 84.8$ & 19.1 & $165.5 \pm 80.3$ & $17.4 \pm 79.5$ & -1.2 \\
\hline $\begin{array}{l}\text { IDL-P } \\
(\mathrm{nmol} / \mathrm{L})\end{array}$ & $37.4 \pm 22.1$ & $54.0 \pm 45.2$ & 53.3 & $60.7 \pm 28.8$ & $27.8 \pm 31.9$ & 24.5 \\
\hline $\begin{array}{l}\text { LDL-P } \\
\text { (nmol/l) } \\
\text { Total } \\
\end{array}$ & $\begin{array}{l}463.6 \pm 246 . \\
7\end{array}$ & $1128.9 \pm 374.6$ & 71 & $985.2 \pm 203.3$ & $332.5 \pm 32.6$ & 21.7 \\
\hline LDL1+2 & $113.5 \pm 40.1$ & $91.4 \pm 81.3$ & 38.33 & $120.4 \pm 45$ & $40.5 \pm 54.1$ & 14.5 \\
\hline $\mathrm{LDL}_{3+4}$ & $80.1 \pm 52.7$ & $119.8 \pm 98.9$ & 57.3 & $129.5 \pm 45$ & $32.5 \pm 67.3$ & -16.7 \\
\hline LDL5+6 & $70.9 \pm 76.8$ & $304.8 \pm 192.1$ & 76.8 & $246.4 \pm 91.8$ & $83.0 \pm 99.4$ & 13.3 \\
\hline $\mathrm{Lp}(\mathrm{a})(\mathrm{nmol} / 1$ & $\begin{array}{l}57.5(5.2,72 . \\
8)\end{array}$ & $\begin{array}{l}15.6 \\
(8.5,29.5)\end{array}$ & 21.1 & $42.6(8.9,62.8)$ & $\begin{array}{l}-13.9(-19.2,- \\
0.3)\end{array}$ & -42.5 \\
\hline ApoB & $42.3 \pm 14.8$ & $67.5 \pm 23.6$ & 60.9 & $71.5 \pm 14.0$ & $21.5 \pm 20.2$ & 20.8 \\
\hline $\begin{array}{l}\text { LDL-C:HDL- } \\
\text { C ratio }\end{array}$ & $0.9 \pm 0.4$ & $2.0 \pm 0.8$ & 67.3 & $1.9 \pm 0.5$ & $0.5 \pm 0.7$ & 18.3 \\
\hline $\begin{array}{l}\text { Apo-B:Apo- } \\
\text { A1 ratio }\end{array}$ & $0.3 \pm 0.1$ & $0.6 \pm 0.2$ & 63.3 & $0.6 \pm 0.1$ & $0.2 \pm 0.2$ & 21.4 \\
\hline LDL-P size & $20.9 \pm 0.5$ & $-0.7 \pm 0.6$ & -3.6 & $20.2 \pm 0.2$ & $0.0 \pm 0.1$ & 0.0 \\
\hline
\end{tabular}

In the experimental group, after a combined therapy of evolocumab and moderate statins for eight weeks, benefits in LDL-C concentrations and other blood lipids measurements were revealed with statistical significance $(\mathrm{P}<0.05)$. TC, LDL-C and its subfractions (LDL-1 to -6$)$, VLDL-C and its subfractions (VLDL-1 to -5$)$, and IDL-C significantly decreased compared to baseline $(\mathrm{P}<0.001)$. The decreased level of LDL-C was significantly attributed to the decrease in the level of small LDL particles (LDL 5+6). In contrast, 
a significant increase in HDL-C was observed $(\mathrm{P}<0.05)$, which could be attributed to an increase in small HDL particles (HDL 3+4). The levels of TC, LDL-C, VLDL-C and IDL-C were reduced by 48.4\%, 65.5\%, $26.3 \%$, and $62.0 \%$, respectively, while the HDL-C level was increased by $7.6 \%$, compared to baseline.

After eight weeks of single moderate statins therapy in the control group, levels of TC, IDL-C, and LDL-C significantly decreased $(\mathrm{P}<0.05)$, by $16.9 \%, 20.7 \%$, and $18.1 \%$, respectively. But the concentration of VLDL-C and HDL-C did not decrease significantly after treatment $(\mathrm{P}>0.05)$.

After eight weeks of treatment, the absolute reduction in the levels of TC, LDL-C, VLDL-C, and IDL-C in the experimental and control groups were $(14.7 \pm 15.4$ vs. $5.2 \pm 12.9),(81.6 \pm 28.7$ vs. $22.9 \pm 25.1),(14.7 \pm$ 15.4 vs. $5.2 \pm 12.9)$, and $(10.0 \pm 7.9$ vs. $4.3 \pm 6.4)$, respectively, with significant differences discerned between the two groups $(\mathrm{P}<0.05)$.

\subsection{Changes in lipoprotein particle concentrations after treatment in the two groups}

Changes in lipoprotein particle concentrations were presented in Table 3. After eight weeks of combined therapy of evolocumab and moderate statins therapy, statistically significant benefits in the concentrations of LDL-P and other lipoprotein particle concentrations were observed $(\mathrm{P}<0.05)$. VLDL-P, IDL-P, LDL-P and its subfractions (LDL-P1 to 6), ApoB and LP(a) all decreased compared to baseline levels $(\mathrm{P}<0.001)$. The concentration of LDL-P before and after medication were $1573.9 \pm 375.3$ vs $463.6 \pm 246.7$ respectively, showing a reduction of $71.1 \%(\mathrm{P}<0.001)$, which could be accounted for by a decrease in small LDL-P (LDL-P5+6). In our study, LDL-P were further classified into large (LDL-P1+2), medium (LDL-P3+4) and small LDL-P (LDL-P5+6) by the size of the particles. The concentrations of small LDL-P decreased by $76.8 \%$, with a significantly larger extent than medium and large LDL-P $(\mathrm{P}<0.001)$. The concentrations of VLDL-P, IDL-P, LP(a), and apoB decreased by $20 \%, 53.3 \%, 21 \%$, and $60.9 \%$, respectively, while the size of LDL-P in the experimental group increased by $3.6 \%(\mathrm{P}<0.001)$, as compared to an insignificant change in the control group.

In the control group, after eight weeks of single moderate statins therapy, IDL-P, LDL-P, and Apo-B concentrations significantly lowered compared to baseline $(\mathrm{P}<0.05)$, but no significant change was found in VLDL-P concentration ( $\mathrm{P}>0.05$ ). The concentrations of IDL-P, LDL-P and ApoB decreased by $24.5 \%$, $21.7 \%$, and $20.8 \%$ compared to baseline. Comparison of LDL-P subfractions revealed no significant change $(\mathrm{P}>0.05)$. LP(a) level was seen no significant decrease but an increase in the control group.

After eight weeks of treatment, the absolute reductions in the concentrations of VLDL-P, IDL-P, LDL-P and ApoB were significantly different between the experimental and control groups $(\mathrm{P}<0.05)$.

\subsection{The achievement of LDL-C treatment target in both groups}

Eight weeks into treatment, the mean LDL-C level in the experimental group decreased from $124 \mathrm{mg} / \mathrm{dl}$ to $44 \mathrm{mg} / \mathrm{dl}$, showing a $65.3 \%$ reduction compared to the baseline, in contrast to a $18.1 \%$ reduction from $104 \mathrm{mg} / \mathrm{d}$ to $81 \mathrm{mg} / \mathrm{dl}$ in the control group. The difference in the percentage of reduction between the two groups was $47.2 \%$, with an absolute difference of LDL-C level of $57 \mathrm{mg} / \mathrm{dl}$.

According to the CCEP Expert Consensus in 2019: for patients at very high risk, the goal of LDL-C control should be lower than $55 \mathrm{mg} / \mathrm{dl}$, or at least a $50 \%$ reduction from baseline. After eight weeks of treatment, $96.3 \%$ patients in the experimental group and $13.3 \%$ in the control group had reached the goal $(\mathrm{P}<0.05)$ (Figure 1). 


\title{
Rate of Lipid Control in Both Groups
}

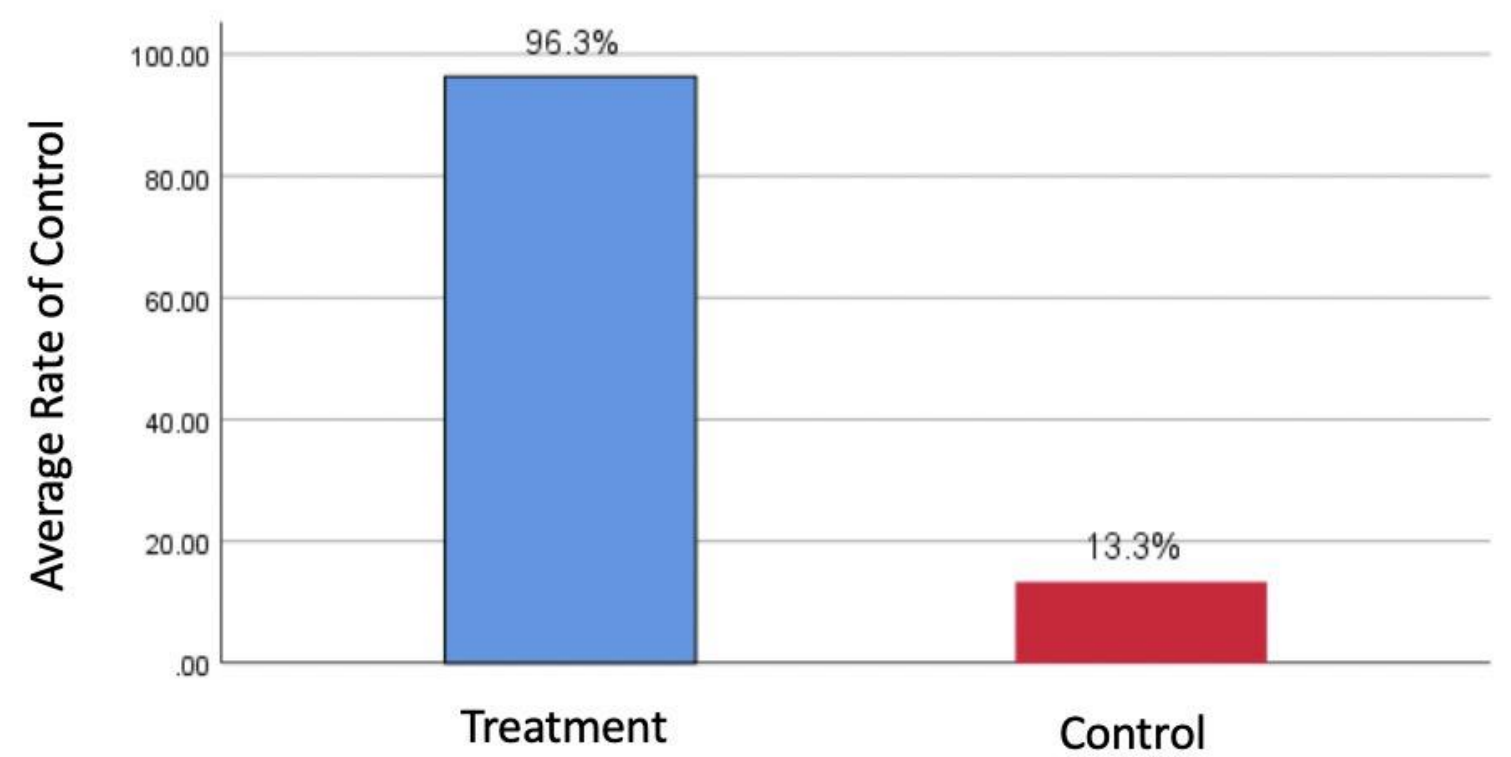

Groups

\begin{abstract}
3. Discussion
PCSK9 inhibitor is a novel mechanism for reducing levels of LDL-C. Evolocumab, a fully human monoclonal antibody targeting human PCSK9, inhibits the binding of PCSK9 and LDL-R. Effective lipid modification could be achieved by PCSK9 inhibitors, manifesting as decreased serum TC, LDL-C, TG, and non-high-density lipoprotein cholesterol, and increased HDL-C ${ }^{[20]}$. In addition, compared to statins, PCSK9 inhibitor exhibit stronger efficacy in lowering LDL-C and TC, as well as increasing HDL-C ${ }^{[21]}$. Compared to statin alone, a combination therapy of PCSK9 inhibitor and statin demonstrates more advantages in lowering LDL-C [22].Although the effect of evolocumab on LDL-C levels is well characterized, little is known about its effects on lipoprotein particles or particle subfractions.
\end{abstract}

It is well-known that lipoprotein particle play an important role in atherosclerosis, including IDL-P, as well as VLDL and LDL-P. More LDL particles could increase the risk of atherosclerosis. Studies found that small LDL particles, which posed a higher threat than larger ones on causing atherosclerosis, were potential predictors of atherosclerosis and coronary artery disease ${ }^{[23]}$, as LDL particles of smaller size and higher density were more difficult to be removed. Smaller size leads to an increased particle density and threedimensional conformation changes of ApoB. From a pure biophysics perspective, smaller LDL particles were more likely to penetrate the vascular endothelial barrier, because they are smaller and denser, and do not require too much subendothelial space during penetration.LDL particles of smaller size and higher density were also more prone to oxidation, and oxidized LDL particles were recognized as the primary target lipoprotein of sub-endothelial phagocytosis for macrophages. As remnant lipoproteins (small molecule VLDL and IDL) are also found in the atherosclerotic plaques, reduction of remnant lipoproteins is also important in addition to the reduction of LDL.

In this study, NMR spectroscopy was adopted to assess the effects of PCSK9 inhibitor (evolocumab) on blood lipid levels and lipoprotein particles subfractions. For the 99 ACS patients with poor lipid control designated to the experimental and control groups, a combination therapy of PCSK9 inhibitor (Repatha ${ }^{\circledR}$, $140 \mathrm{mg}$, q2w) and moderate statin (rosuvastatin, $10 \mathrm{mg}$, qn), or moderate statin therapy (rosuvastatin, 10mg, qn) alone was administered. The lipid profiles and lipoprotein particles subfractions were measured by NMR spectroscopy after eight weeks of medication, and the achievement of LDL-C treatment target in both groups was also analyzed.

In this study, the two groups of patients were well-matched in terms of demographic features, clinical characteristics, baseline blood lipid levels, and lipoprotein particles concentrations.After eight weeks of 
treatment, the TC, LDL-C, VLDL-C and IDL-C levels were decreased by $48.4 \%, 65.5 \%, 26.3 \%$, and $62.0 \%$ on average in the experimental group, respectively. The HDL-C level was increased by $7.6 \%$. ApoB, representing the level of circulating numbers of atherogenic lipoproteins, decreased by $60.9 \%$. These revealed significantly different lipid profiles compared to baseline. The decreased level of LDL-C was attributed to decreased small LDL particles (LDL5+6), and the reduction of VLDL-C was due to that of small VLDL particles (VLDL4+5). Eight weeks later, the absolute reductions in TC, LDL-C, VLDL-C and IDL-C level in both the experimental and control groups were $(14.7 \pm 15.4$ vs. $5.2 \pm 12.9),(81.6 \pm 28.7$ vs. $22.9 \pm 25.1),(14.7 \pm 15.4$ vs. $5.2 \pm 12.9)$, and (10.0 \pm 7.9 vs. $4.3 \pm 6.4)$, respectively, exhibiting differences of statistical significance $(\mathrm{P}<0.05)$. Thus, the combination therapy showed significant advantages over statins alone.

In terms of lipoprotein particles subfractions, after eight weeks of treatment, the experimental group showed statistically significant benefits in LDL-P concentration and other lipoprotein particles concentrations $(\mathrm{P}<$ 0.05). VLDL-P, IDL-P, LDL-P and its subfractions (LDL-P1 to 6), and LP(a), were significantly decreased compared to baseline $(\mathrm{P}<0.001)$. The concentration of LDL-P was reduced by $71.1 \%$ compared to baseline $(\mathrm{P}<0.001)$, which could be mainly attributed to the reduction in small LDL-P (LDL-P5+6). The level of small LDL-P decreased by $76.8 \%$, to a significantly larger extent compared to medium and large LDL-P (P $<$ 0.001). Levels of VLDL-P, IDL-P, and LP(a) decreased by $20 \%, 53.3 \%$, and $21 \%$, respectively. After eight weeks of treatment, the absolute reductions of which in the experimental group were also more prominent compared with the control group, with statistical significance $(\mathrm{P}<0.05)$.

It is well accepted that smaller size of lipoprotein particles leads to an increased risk for atherosclerosis ${ }^{[24,25]}$. In our study, evolocumab exhibited significant advantages in lowering the levels of small lipoprotein particles. After further classifying LDL and VLDL into small, medium, and large particles, we found a significant larger reduction in small LDL (LDL5+6) and VLDL (VLDL4+5), compared to medium and large particles. Meanwhile, the size of LDL-P before and after medication in the experimental group were $20.2 \pm$ 0.4 vs $20.9 \pm 0.5$, respectively, suggesting an increase of $3.6 \%$ in the size of lipoproteins after treatment with evolocumab. The FOURIER trial found that evolocumab could reduce the incidence of primary endpoint event by $15 \%$, which might be associated with a decrease of particles which could result in atherosclerosis., in addition to lower levels of LDL-C.

2019 CCEP Expert Consensus recommended that the goal of lipid control for recent ACS patients , a super high risk population of ASCVD, should achievement: an LDL-C level of less than 55mg/dl, or a reduction of LDL-C of at least $50 \%$ compared to baseline ${ }^{[26]}$. In this study, the treat-to-target rate of blood lipids with statin alone was as low as $13.3 \%$. ACS patients have a very high risk of early cardiovascular, with over $30 \%$ of cardiovascular events and mortality occurring in the first four days, and over $50 \%$ occurring in the first 15 days ${ }^{[27]}$. Previous studies showed that the major cause of early ACS risks was the rupture of atherosclerotic plaques ${ }^{[28]}$. Therefore, the 2019 ESC Guidelines for the Management of Dyslipidemias recommended that : for patients who present with an ACS and whose LDL-C levels are not at goal, despite already taking a maximally tolerated statin dose and ezetimibe, the addition of a PCSK9 inhibitor early after the event (during hospitalization for the ACS event if possible) should be considered, in order to obtain earlier cardiovascular benefits ${ }^{[29]}$.

There were some limitations in this study. The sample size was relatively small, and the duration was relatively short, making it difficult to evaluate the definitive clinical outcomes. Thus, future studies with larger sample size and longer follow-up period are needed to evaluate the effects of medications on clinical events and outcomes. In conclusion, eight weeks of PCSK9 inhibitor could significantly improve the plasma lipid profiles in ACS patients with poor lipid control, and significantly decrease the atherogenic lipoproteins particles including LDL-P and remnant lipoproteins particles. Given the low treat-to-target rate of blood lipids in ACS patients on statin monotherapy, an additional PCSK9 inhibitor is recommended to be initiated as early as possible to obtain earlier cardiovascular benefits.

\footnotetext{
Abbreviations

NMR:Nuclear magnetic resonance;ACS:Acute coronary syndromes;PCSK9:Proprotein convertase subtilisin/kexin type 9;ASCVD:Atherosclerotic cardiovascular disease ;BMI: Body Mass Index ;STEMI :Stsegment Elevation Myocardial Infarction ;NSTEMI :non-ST-segment elevation myocardial infarction ; UAP:Unstable Angina Pectoris;LDL-C:low-density lipoprotein
} 
cholesterol;IDL-C:intermediate-density lipoprotein;VLDL-C :very low-density lipoprotein cholesterol; HDL-C:high-density lipoprotein cholesterol; Lp(a):Lipoprotein a; ApoB:Apolipoprotein B;LDL-P:lowdensity lipoprotein particle concentration;VLDL-P: very low-density lipoprotein particle concentration;IDLP : intermediate-density lipoprotein particle concentration; Q1, Q3 : fifirst and third quartiles; SD : standard deviation.

\section{Acknowledgements}

None.

\section{Authors' contributions}

Hongliang Cong made contributions to the conception and design of the study. Tingting Li collected blood samples, analyzed the data, wrote the manuscript and drew the figures; Yiying Zhang also analyzed some data and collected the information of the subjects.All the authors have accepted responsibility for the entire content of this submitted manuscript and approved submission

\section{Funding}

None.

\section{Availability of data and materials}

The datasets used and/or analysed during the current study are available from the corresponding author on reasonable request.

\section{Ethics approval and consent to participate}

This study was approved by the Medical Ethics Committee of Tianjin Chest Hospital.Informed consents were obtained from all subjects.

\section{Consent for publication}

Not applicable.

\section{Competing interests}

The authors declare that they have no competing interests 


\section{References}

[1] Yokoyama K,Tani S,Matsuo R,et al.Increased triglyceride /high- density lipoprotein cholesterol ratio may be associated with reduction in the low-density lipoprotein particle size: assessment of atherosclerotic cardiovascular disease risk[J].Heart Vessels,2019,34( 2) : 227-236.

[2] Lyons JG,O'Dea K, Walker KZ. Evidence for low high-density lipoprotein cholesterol levels in Australian indigenous peoples: a systematic review[J], BMC Public Health,2014,14: 545.

[3]Cromwell WC, Otvos JD, Keyes MJ, Pencina MJ, Sullivan L, Vasan RS, Wilson PW, D'Agostino RB.

LDL Particle Number and Risk of Future Cardiovascular Disease in the Framingham Offspring StudyImplications for LDL Management. J Clin Lipidol. 2007; 1:583-592.

[4]Otvos JD, Mora S, Shalaurova I, Greenland P, Mackey RH, Goff DC Jr. Clinical implications of discordance between low-density lipoprotein cholesterol and particle number. J Clin Lipidol. 2011;5:105-113. [5]Packard CJ. Determinants of achieved LDL cholesterol and "nonHDL" cholesterol in the management of dyslipidemias. Curr Cardiol Rep. 2018;20:60.

[6]Sandesara PB, Virani SS, Fazio S, Shapiro MD. The forgotten lipids: triglycerides, remnant cholesterol, and atherosclerotic cardiovascular disease risk. Endocr Rev. 2019;40:537-57.

[7]Langlois MR, Chapman MJ, Cobbaert C, Mora S, Remaley AT, Ros E, et al. European Atherosclerosis Society (EAS) and the European Federation of Clinical Chemistry and Laboratory Medicine (EFLM) Joint Consensus Initiative. Quantifying atherogenic lipoproteins: current and future challenges in the era of personalized medicine and very low concentrations of LDL cholesterol. A Consensus Statement from EAS and EFLM. Clin Chem. 2018;64:1006-33

[8] Goliasch G,Oravec S,Blessberger H,et al.Relative importance of different lipid risk factors for the development of myocardial infarction at a very young age ( $\leq 40$ years of age) $[\mathrm{J}]$. Eur J Clin Invest, 2012,42(6):631-636.

[9] Cole TG, Contois JH, Csako G, McConnell JP, Remaley AT, Devaraj S, Hoefner DM, Mallory T, Sethi AA, Warnick GR. Association of apolipoprotein B and nuclear magnetic resonance spectroscopy-derived LDL particle number with outcomes in 25 clinical studies: assessment by the AACC Lipoprotein and Vascular Diseases Division Working Group on Best Practices. Clin Chem. 2013;59:752-770.

[10]Mora S, Buring JE, Ridker PM. Discordance of low-density lipoprotein (LDL) cholesterol with alternative LDL-related measures and future coronary events. Circulation.2014;129:553-561.

[11]Otvos JD, Mora S, Shalaurova I, Greenland P, Mackey RH, Goff DC Jr. Clinical implications of discordance between low-density lipoprotein cholesterol and particle number. J Clin Lipidol. 2011;5:105-113. [12] Sabatine MS,Giugliano RP,Keech AC,et al.Evolocumab and clinical outcomes in patients with cardiovascular disease[J].N Engl J Med,2017,376:1713-1722.

[13]Nicholls SJ,Puri R,Anderson T, et al. Effect of evolocumab on progression of coronary disease in statintreated patients.The GLAGOV Randomized Clinical Trial [J]. JAMA, 2016, 316(22): 2373-2384.

[14]Jeyarajah EJ, Cromwell WC, Otvos JD. Lipoprotein particle analysis by nuclear magnetic resonance spectroscopy. Clin Lab Med 2006;26:847- 870.

[15]Jimenez B, Holmes E, Heude C, et al. Quantitative lipoprotein subclass and low molecular weight metabolite analysis in human serum and plasma by $1 \mathrm{~h} \mathrm{nmr} \mathrm{spectroscopy} \mathrm{in} \mathrm{a} \mathrm{multilaboratory} \mathrm{Trial[J].}$ Analytical Chemistry, 2018, 90(20): 11962-11971.

[16] Dona AC, Jiménez B, Schäfer H, Humpfer E, Spraul M, Lewis MR, Pearce JTM, Holmes E, Lindon JC, Nicholson JK. Precision high-throughput proton NMR spectroscopy of human urine, serum, and plasma for large-scale metabolic phenotyping. Anal Chem. 2014; 86:9887-94.

[17]Jimenez B, Holmes E, Heude C, Tolson RF, Harvey N, Lodge SL, Chetwynd AJ, Cannet C, Fang F, Pearce JTM, et al. Quantitative lipoprotein subclass and low molecular weight metabolite analysis in human serum and plasma by H-1 NMR spectroscopy in a multilaboratory trial. Anal Chem. 2018;90(20): 11962-71. [18]Harbaum L, Ghataorhe P, Wharton J, Jimenez B, Howard LSG, Gibbs JSR, Nicholson JK, Rhodes CJ, Wilkins MR. Reduced plasma levels of small HDL particles transporting fibrinolytic proteins in pulmonary arterial hypertension. Thorax. 2019;74(4):380-9.

[19] Okazaki M, Usui S, Ishigami M, Sakai N, Nakamura T, Matsuzawa Y, Yamashita S. Identification of unique lipoprotein subclasses for visceral obesity by component analysis of cholesterol profile in highperformance liquid chromatography. Arterioscl Throm Vas. 2005;25(3):578-84.

[20]Sabatine, M. S. et al. Evolocumab and clinical outcomes in patients with cardiovascular disease[J]. N. Engl. J. Med, 2017, 376, 1713-1722. 
[21] Roth EM, Taskinen MR, Ginsberg HN, et al. Monotherapy with the PCSK9 inhibitor alirocumab versus ezetimibe in patients with hypercholesterolemia: results of a 24 week, double-blind, randomized Phase 3 trial[J]. Int J Cardiol, 2014, 176(1):55-61.

[22]Koren MJ, Lundqvist P, Bolognese M, et al. Anti-PCSK9 monotherapy for hypercholesterolemia: the MENDEL-2 randomized, controlled phase III clinical trial of evolocumab[J]. J Am Coll Cardiol, 2014, 63(23):2531-2540.

[23]Williams PT,Zhao XQ,Marcovina SM,et al. Levels of cholesterol in small LDL particles predict atherosclerosis progression and inci-dent CHD in the HDL-Atherosclerosis Treatment Study (HATS) [J] . PLoS One,2013,8( 2) : e56782.

[24]Krauss RM. All low-density lipoprotein particles are not created equal. Arterioscler Thromb Vasc Biol. 2014;34:959-61.

[25] Mora S, Caulfield MP, Wohlgemuth J, Chen Z, Superko HR, Rowland CM, Glynn RJ, Ridker PM, Krauss RM. Atherogenic lipoprotein subfractions determined by ion mobility and first cardiovascular events after random allocation to high-intensity statin or PlaceboCLINICAL PERSPECTIVE. Circulation. 2015;132:2220-9.

[26]2019 Expert recommendations of the China Cholesterol Education Program(CCEP) for lipid-lowering therapy to reduce cardiovascular events [J].Chinese Journal of Internal Medicine,2020(01):18-2.

[27] Fox KA, Frederick A,Goodman,SG,et al.Time course of events in acute coronary syndromes:implications for clinical practice from the GRACE registry[J].Nat Clin Pract Cardiovasc Med,2008,5(9):580-589.

[28]Gallone G, Baldetti L, Pagnesi M, et al. Medical Therapy for Long-Term Prevention of Atherothrombosis Following an Acute CoronarySyndrome: JACC State-of-the-Art Review[J]. J Am Coll Cardiol, 2018, 72(23 Pt A): 2886-2903.

[29]Mach F, Baigent C, Catapano A L, et al. 2019 ESC/EAS Guidelines for the management of dyslipidaemias: lipid modification to reduce cardiovascular risk[J]. Eur Heart J, 2019. 
Figures

\section{Rate of Lipid Control in Both Groups}

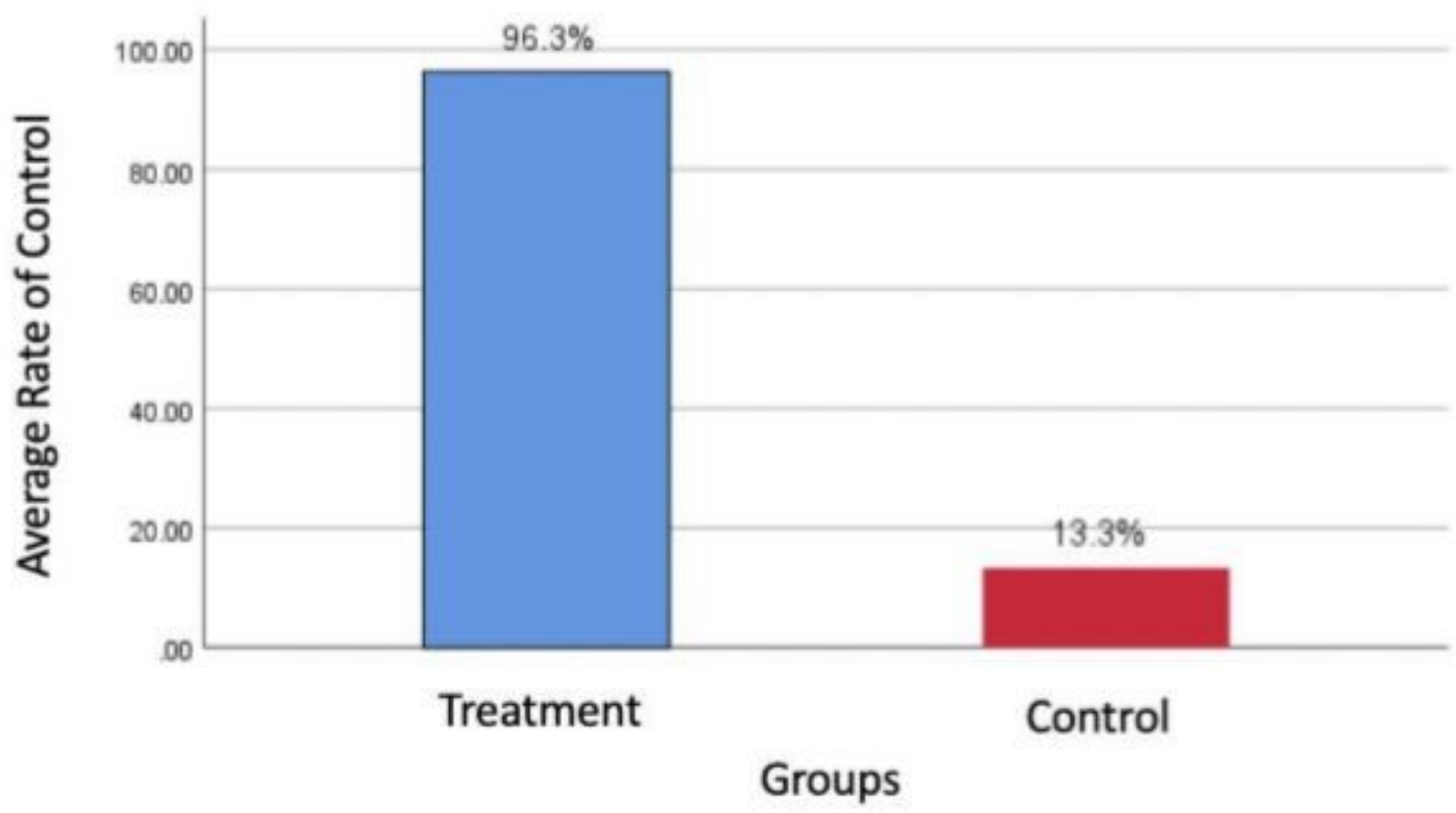

Figure 1

Fig 1 